\title{
Structural complexity in riverine and terrestrial habitat networks affects population abundance and diversity
}

\author{
$\underline{\text { J.A. Webb }}^{\text {a }}$, M. Padgham ${ }^{\text {ab }}$ \\ a $e$ Water Cooperative Research Centre \& Deptartment of Resource Management \& Geography, \\ University of Melbourne, Australia \\ ${ }^{\mathrm{b}}$ Water Studies Centre, Monash University, Australia \\ Email: angus.webb@unimelb.edu.au
}

\begin{abstract}
Landscape structure is an important determinant of spatial variation in population and community processes. Theoretical research commonly assumes that extinction and recolonisation can be modelled without considering population dynamics, and ignores the latter in metapopulation models. However, the scales at which landscapes must be actively managed are generally commensurate with scales of population dynamics, suggesting the importance of developing models of (meta-)population response that explicitly consider population dynamics.
\end{abstract}

It has been previously demonstrated that habitat network structure affects the responses of metapopulations in two-dimensional habitat networks typical of terrestrial systems. However, other types of habitat networks have largely been ignored. In particular, river systems follow a rigid hierarchical branching structure to form a Dendritic Ecological Network (DEN), which differs fundamentally from terrestrial systems because habitat patches are only ever linked by a single path. It has been hypothesized that this unique structure must have important implications for resident populations and communities.

We developed a theoretical method to relate both population abundance and two different measures of population diversity to landscape structure. We simulated lattice and dendritic networks of differing complexity, representing open terrestrial landscapes with no particular constraints on patterns of connectivity, and river networks constrained to bifurcating connectivity patterns, respectively. We used two different measures to quantify structural complexity. Landscapes were simulated as directed graphs, with nodes representing individual habitat patches defined by a habitat quality or carrying capacity. Patches were connected by directed graph edges, with asymmetrical edge weights quantifying the probability of organisms being able to move in each direction between pairs of nodes. Density dependent population processes were modelled stochastically within individual patch-level populations.

Results were affected both by the way in which structural complexity was represented, and also by the way in which diversity was quantified. Nevertheless, both the type and the complexity of the habitat network affected population abundance and diversity. Complex lattice and dendritic networks supported the highest and lowest abundances, respectively. As complexity was reduced, network-scale carrying capacities of the two network types converged. The results for diversity were quite different. Diversity was almost unaffected by structural complexity in dendritic networks, and was higher than for any lattice network. Within lattice networks, simple structures had the highest diversity. Simple lattice networks are structurally similar to complex dendritic networks, except for the occasional presence of loops and multiple paths. These features appear to have profound effects on network-scale abundance and diversity.

These results show that the unique structure of dendritic ecological networks do indeed have unique implications for resident organisms. They suggest that highly branched riverine systems are more able to foster diverse ecosystems that less branched equivalents and terrestrial landscapes. They also call into question the predominate use of metapopulation models that ignore population processes when studying the effects of habitat network structure.

Keywords: Landscape structure, lattice network, dendritic ecological network (DEN), complexity, population, abundance, diversity 


\section{INTRODUCTION}

The abundance and diversity of biological populations are determined by processes across broad spatial and temporal scales (Levin, 2000). Diversity is, however, most readily recognised through comparison between populations, whether of different species, different regions, or both (MacArthur, 1957). This implies that there must be minimal spatial and temporal scales across which diversity may be defined. Because variations in relative abundance are more easily measured in space than time, most attempts to quantify diversity have examined spatial variation (Whittaker, 1972). This 'beta' component of diversity (Sørensen, 1948) is generally quantified from differences in species or genetic composition between spatially separated communities or populations (e.g. Kessler, 2009).

Diversity is largely determined through extinction, speciation, and movement. Extinction and speciation operate over long temporal scales, but movement operates over short temporal scales for most species. This disparity forces a choice between examining extinction and speciation over paleontological scales (e.g. Sahney et al., 2010), or examining the spatial aspect of diversity at the expense of more detailed examination or understanding of extinction and speciation (e.g. Gaston et al., 2008). As a consequence, empirical studies of diversity are dominated by spatial comparisons, while theoretical studies are equally dominated by temporal comparisons of rates of extinction and species turnover.

In both cases, it is convenient to conceive of diversity at scales sufficiently large such population dynamics may be ignored (Drechsler and Wissel, 1997). A single-species community modelled at such scales is conceived of as a metapopulation (Levins, 1969). For metapopulations, diversity is determined by processes analogous to extinction and speciation, namely local extirpation and (re)colonisation (Hanski, 1998). A metapopulation approach to diversity removes any consideration of population abundances as habitat patches are simply considered as either occupied or unoccupied. Instead, diversity in metapopulation models is commonly quantified in terms of rates of species persistence or turnover (e.g. Hanski and Ovaskainen, 2000). While such notions of diversity have provided much insight to similarly scaled patterns of species richness and abundance (McGill, 2010), ongoing management of natural landscapes and their communities requires considering diversity at fundamentally smaller and shorter scales. It is possible that at such scales, the assumption that population processes can be ignored may not be valid.

Diversity is examined here both as a spatial pattern that exists at every instant, and also as a temporally evolving process. The latter case corresponds to the previously-mentioned theoretical approach of examining species turnover, and is defined simply through a probability of extinction, $p_{e}$. If landscape-scale processes of extinction and colonisation can be assumed independent of population processes (sensu Hanski, 1998), differences in metapopulation responses to different networks should depend only upon the spatial arrangement of habitat patches and connectivity relationships among them (1.e. network topography). Thus, metapopulation-scale extinction and recolonisation-and therefore diversity-should be quantifiable directly from a matrix $\mathbf{p}$ that quantfies the interactions between habitat patches. However, as noted above, since a metapopulation view of large-scale population processes has predominated in the literature, there has been little examination of how habitat network topography affects population processes, including spatial diversity in abundance, and overall abundance itself.

Habitat networks may be depicted as graphs (Urban et al., 2009), were the nodes represent patches of habitat and the edges represent connections between those patches. Such models have been increasingly used in landscape ecological studies in recent years (Urban et al., 2009). A two-dimensional terrestrial landscape manifests as a lattice network, where habitat patches are arranged with no particular structure, and are connected by multiple potential pathways along which movement may occur. However, other types of habitat networks also exist. In particular, river systems are characterised by rigidly hierarchical topographic structures in which linear habitat patches converge in one direction only (i.e. downstream), and within-stream dispersal among habitat patches is constrained to a single path. This particular configuration, which has since been recognised as existing in other landscapes (e.g. cave networks, animals that live in individual trees, hedgerows), is referred to as a Dendritic Ecological Network (DEN; Grant et al., 2007). This fundamentally different structure of dendritic landscapes has led to speculation that the biotas of river systems may face very different constraints compared to those of terrestrial systems (Wiens, 2002). However, no studies have directly compared population processes between terrestrial and dendritic networks. 
Here, we measure the impact of network topography on population abundance, probability of extinction, and diversity. We also compare the topographical effects of lattice and dendritic networks, thereby testing whether effects of network topography are transferable among different types of landscapes.

\section{METHODS}

\subsection{Simulations}

Network Simulations. Lattice networks were simulated on five-by-five node square grids by randomly adding edges until all nodes were connected together. Complexity of the network was determined by the number of edges required to connect all the nodes. Dendritic networks of 25 nodes were simulated from the most downstream node upwards, branching according to defined probabilities. Higher branching probabilities produced more complex networks. Networks were randomly generated until each possible combination of the values of the two measures of network topography (see below) had been produced at least 10 times. In all, just over 3000 individual network structures were generated.

Landscape Matrices. Landscape matrices, $\mathbf{p}$, were constructed from transition probabilities between all pairs of nodes, as described in Padgham and Webb (2010). Briefly, each node was defined by a quality $(0<q<1)$, and each edge joining a pair of nodes by a pair of asymmetrical (i.e. node $i$ to node $j$ and vice-versa) connectivities $(0<c<1)$ quantifying the relative likelihood or ease of passage between those two nodes. In a simple, linear network with connectivities, $c_{i}$ connecting node $i$ to node $i+1$, transition probabilites between nodes $i$ and $j$ are,

$$
p_{i j}=\frac{q_{j} \prod_{k=i}^{j-1} c_{k}}{\sum_{k}\left(q_{k} \prod_{m=i}^{k-1} c_{m}\right)},
$$

with transition probabilites for more complex networks simply requiring more complex summations.

Values of $q$ and $c$ were respectively assigned to each network node and edge according to a fixed probability distribution of $\exp \left[-(x-0.5)^{2} / 0.2^{2}\right]$, which approximates a truncated (at 0,1$)$ normal distribution. For each randomly-generated network structure, the same values of $q$ and $c$ were randomly re-allocated to nodes and edges 50 times. This allowed us to study the effects of network structure on abundance and diversity more so than the effects of the spatial distributions of $q$ and $c$ values. The results below are thus based on more than 150,000 simulations.

Population Simulations. Populations were simulated with a sigmoidal growth function (Boukal and Berec, 2002), $g(N)=(\rho / q)(1-N / q)\left(N-N_{0}\right)$, where $\rho$ determines the rate of population dynamics, $q$ is the carrying capacity (i.e. the habitat quality), and a value of $N_{0}=0.1$ was used throughout to define a threshold below which growth rates are negative. Environmental stochasticity was introduced through imposing Gaussian noise on the parameter, $q$, such that $q \sim q_{0}+N(0, \sigma)$, with $\sigma=0.1$ used throughout (Drechsler and Wissel, 1997).

Populations were run with fixed $q$ until convergence, following which stochastic noise was introduced, and means and standard deviations of abundance and diversity calculated over a subsequent 1,000 iterations. Movement was scaled to the same rate as population dynamics, through scaling the landscape matrix, $\mathbf{p}$, by a rate of movement, $\mu=\rho=0.1$, such that $\left.p_{i j}^{\prime}\right|_{j \neq i}=\mu p_{i j}$, and $p_{i i}^{\prime}=1-\mu\left(1-p_{i i}\right)$.

\subsection{Metrics}

Measures of Network Topography. We related both abundance and diversity to two measures of complexity: the network radius, $r$ (Diestel, 2005), and the 'Nodal Isolation', N.I. (Figure 1). Complex networks are characterised by low values for both $r$ and N.I. To provide unibiased estimates of the dependence of abundance and diversity upon these structural parameters, the simulation programs were constructed to ensure that each network represented a unique combination of $r$ and N.I.

Abundance. We measured network-scale abundance $N$ simply as the sum of abundances across the 25 nodes. The sigmoidal population growth function was scaled such that each node had a theoretical maximum population (carrying capacity) of 1.0 if $q=1$. 

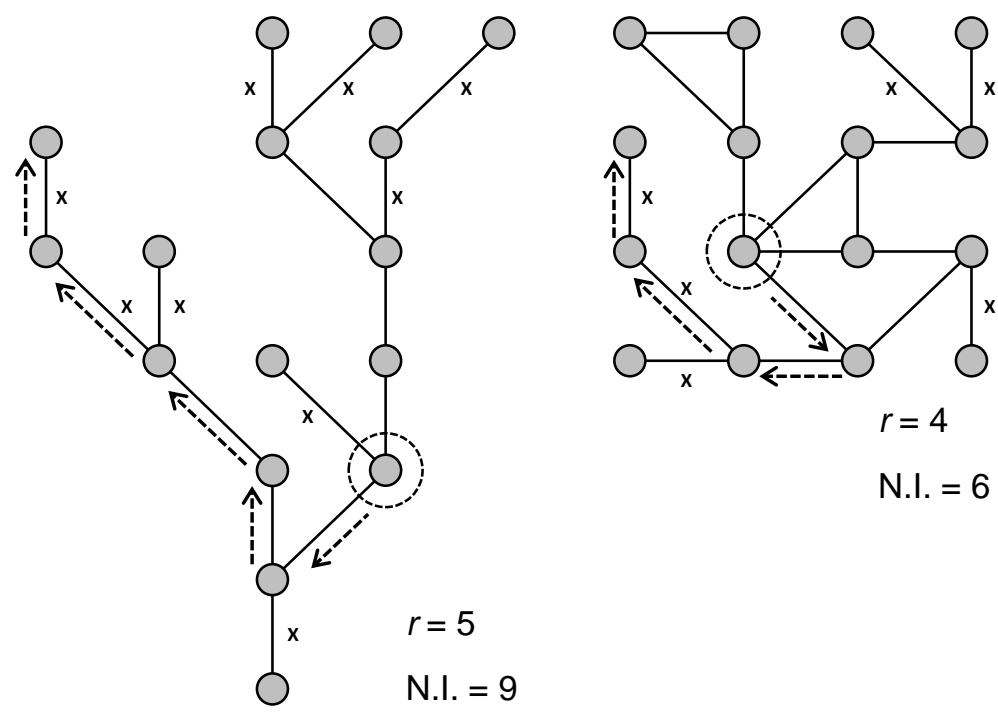

N.I. $=6$

Figure 1. Measures of network topography for dendritic and lattice networks. Filled circles are nodes, lines between them are edges. Network radius $(r)$ is defined as the miniumum eccentricity (the shortest distance from a node to the node most distant in the network) for the network (Diestel, 2005), illustrated by dotted arrows leading away from the 'central' (circled) node. Nodal Isoluation (N.I.) is the summed number of edges traversed from all terminal nodes until the first branching node is encountered, illustrated by ' $\mathrm{x}$ ' next to those edges that would be counted.

Probability of Extinction. The probability distribution of abundance, $p(N)$, responds to stochastic environmental changes, as $p_{e}=\int_{-\infty}^{0} p(N) d N$. With Gaussian environmental noise of standard deviation $\sigma$, this translates to $p_{e}=(1 / 2)[1+\operatorname{erf}(-\bar{N} /(\sigma \sqrt{2}))]$, where $\bar{N}$ is the first moment of the probability density of abundance, $N \int_{0}^{-\infty} p(N) d N$. These probabilities of extinction are directly equivalent to rates of species turnover, as is commonly assumed in metapopulation modelling.

Diversity of Movement. The spatial pattern of diversity was quantified through calculating the diversity of movement throughout a network. In terms of transition probability or diffusion matrices, $\mathbf{p}$, and nodal abundances, $\mathbf{N}$, the diversity of movement between two nodes $A$ and $B$ in a network of $n$ nodes is,

$$
D_{A B}=\sum_{i}\left|\frac{N_{i} p_{i A}}{N_{A}}-\frac{N_{i} p_{i B}}{N_{B}}\right|=\sum_{i} N_{i}\left|\frac{p_{i A}}{N_{A}}-\frac{p_{i B}}{N_{B}}\right|,
$$

with the average over an entire network being,

$$
D_{M}=\frac{2}{n(n-1)} \sum_{i=1}^{n-1} \sum_{j=i+1}^{n} D_{i j}
$$

Diversity of movement is equivalent to diversity in the distributions of $p_{i j}$ values (Eq. 2), with high values arising in networks with more variable qualities, connectivities, or both.

\section{RESULTS}

While the most complex lattice networks supported the greatest abundance, the greatest abundances in dendritic networks were in the simplest networks (Figure 2a). Responses to N.I. reconciled these apparently opposing tendencies, with both networks aligning along a common response such that abundance 
increased approximately linearly as N.I. increased or decreased away from 14 (Figure 2d). While abundances in dendritic networks at either extreme (N.I. $=4$ or 24 ) were approximately equal, the continuation at the lower end with lattice networks suggests that dendritic networks that support the highest abundances are those with large $r$ and low N.I. The simplest dendritic network is a straight line which has the largest $r$, yet the largest N.I. (equal to the number of nodes). In contrast, a network with branches just prior to the two terminal nodes of an otherwise linear system has N.I. $=4$ and $r=(n-1) / 2=12$.
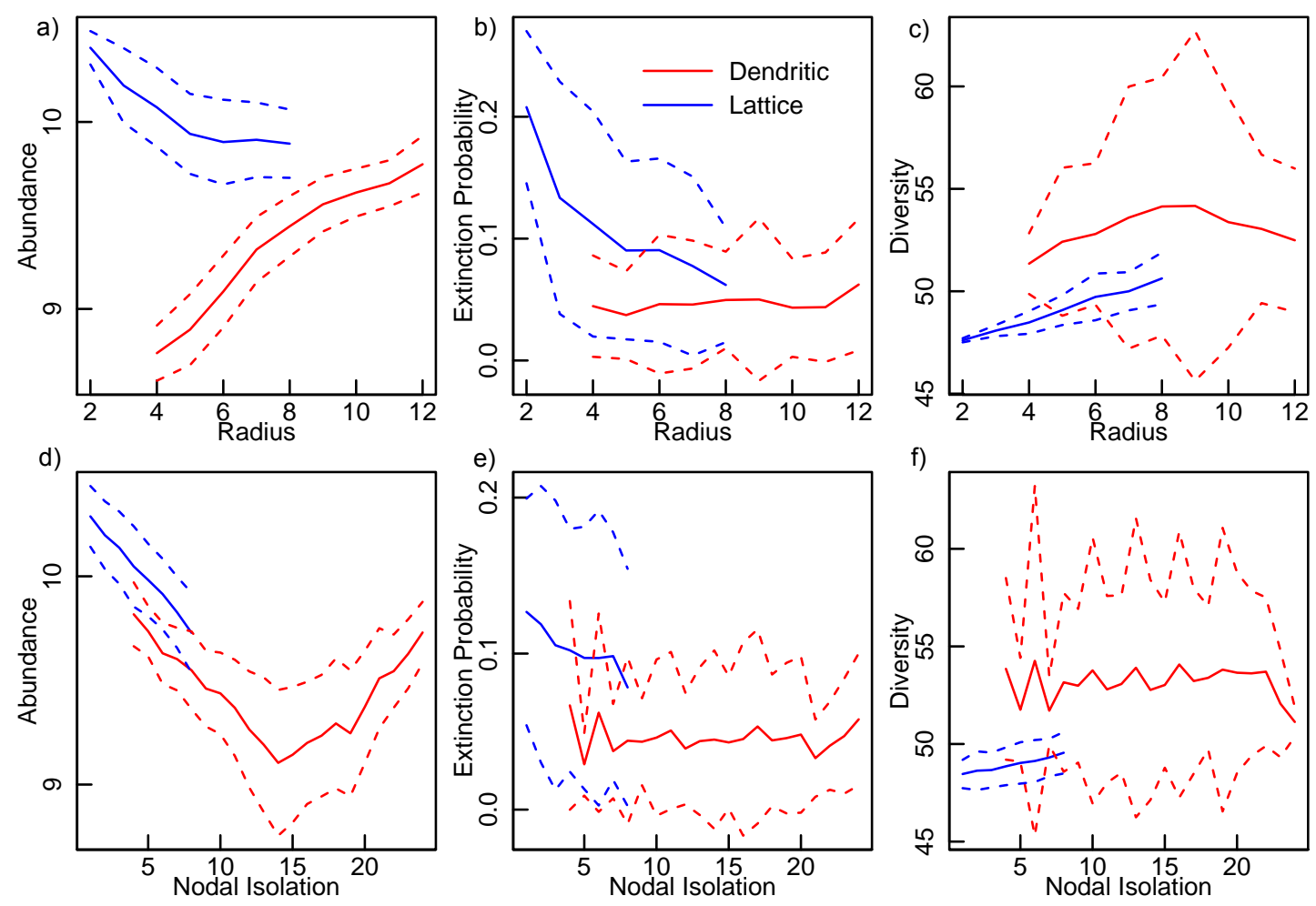

Figure 2. Dependence of population Abundance (a, d), Probability of Extinction (b, e), and Diversity of Movement (c, f) on network Radius (a, b, c) and Nodal Isolation (d, e, f). Blue and red lines are mean \pm 1 $\mathrm{SD}$ response for all simulated lattice and dendritic networks, respectively.

Lattice networks with no internal loops, which will generally have large radii, are structurally equivalent to dendritic networks. Importantly, it is at this extreme that the trajectories for abundance in lattice networks converged with those for dendritic networks. Thus, the presence of internal loops in lattice networks increased abundance beyond that of any dendritic network. The networks supporting the highest abundance were fully-connected lattices of minimal $r$ and N.I., in which every node had multiple edges.

Such networks, however, had much lower diversity of movement (average $D_{M}=49.0$ for lattice and 53.3 for dendritic, $p<0.001$ ). Moreover, while simpler (more dendritic-like) lattice networks had higher values of $D_{M}$ that approached those of dendritic networks, diversity in dendritic networks was independent of network structure (structure explained less than $2 \%$ of the observed variance; Figure $2 \mathrm{c}$, f). Responses of diversity to network structure of both network types thus suggest that diversity of movement generally remains independent of structure as long as there is only one path between any pair of nodes — the characteristic property of dendritic networks. The multiple paths that arise in lattice networks significantly decrease diversity of movement throughout a network, an intuitive result given that multiple paths offer greater opportunity for movement amongst nodes and resultant decreased diversity.

Probabilities of nodal extinction generally reversed these patterns for movement diversity, such that dendritic networks had structurally independent, constant extinction probabilities. Within lattice networks, the simplest, most dendritic-like networks had the lowest extinction probabilities (Figure 2b, e). 


\section{DISCUSSION}

\subsection{The importance of network type and complexity}

This work has clearly shown that the spatial structure of habitat networks has marked effects on population processes. Both abundance and two different measures of diversity responded to differences in the spatial arrangement and connectivities of habitat patches. These effects were sometimes substantial. Population processes in the two different types of networks (lattice and dendritic) responded quite differently to differences in structural complexity. For abundance, the two network types responded in opposite directions to change in complexity. For the two measures of diversity, lattice networks showed a response to changes in network complexity, dendritic networks did not.

Thus, it does appear that dendritic networks are fundamentally different habitat networks compared to the open two-dimensional lattices characteristic of terrestrial systems. The absence of loops and multiple paths within dendritic ecological networks leads to systems more likely to have habitat patches that are relatively isolated from the remainder of the network. Such a spatial arrangement could well be expected to support higher between-patch diversity. Conversely, it is reasonable to hypothesize that higher carrying capacities and lower diversity of terrestrial systems may have driven evolutionary forces to select for organisms with superior competitive abilities in these type of environments. However, the vastly different disturbance regimes that characterise terrestrial and river systems makes an empirical comparison of the two network types extremely difficult.

\subsection{Choice of metrics}

The apparent effects of structural complexity on population processes depend at least partly on the measure used to quantify complexity. While network radius is widely used as a measure of complexity in graph theory (Diestel, 2005), it fails to capture many details. In particular, when network complexity is quantified as Nodal Isolation, the two network types form more of a continuum. The most complex dendritic networks have similar levels of nodal isolation as the simplest lattice networks, and both abundances and diversities for lattice and dendritic networks of equivalent N.I. were similar. The slight differences between the two network types at these overlapping levels of nodal isolation is probably attributable to the presence of loops in lattice networks.

The two different measures of diversity also demonstrate the importance of the choice of metric on the results and their interpretation. As a general pattern, probabilities of extinction were inversely related to movement diversity - an intuitively sensible result. However, probabilities of extinction showed far greater relative uncertainty at any given level of structural complexity, but only for lattice networks, a result for which we have no explanation as yet. Metapopulation models generally only use probability of extinction as a measure of diversity, which may reduce the insight that can be gained from them.

\subsection{Populations and metapopulations}

By themselves, these results do not invalidate the metapopulation view that effects of habitat structure can be explored while ignoring population processes. It is possible that classical metapopulation models run over the same set of networks would give similar results for probabilities of extinction as observed here. However, the effects of network structure on those metrics that relate explicitly to population processes - abundance and movement diversity — demonstrate that a richer understanding of the effects of habitat structure may be gained through a spatially explicit population modelling approach. The result that network type and structural complexity affects network-scale abundance (i.e. carrying capacity) is of particular interest since it could not have been observed using a metapopulation modelling approach.

As stated above, management of natural environments may require consideration of habitat networks at finer spatial and temporal scales than are assumed in metapopulation models. The techniques used in this study may offer greater relevance at these smaller scales. Finally, given that many landscapes manifest scale-free topographical properties over wide spatial ranges (Sayles and Thomas, 1978), individual nodes of a simulated network can be considered as either sites of local populations, or aggregated metapopulations, with the dependence on network topography being the same in both cases. Such a conception calls into question the idea that ecological dynamics may be studied without considering population processes. 


\section{CONCLUSIONS}

The spatial arrangement of habitat patches affects both the diversity and abundance of resident organisms. Lattice networks typical of terrestrial habitat networks respond qualitatively differently to changes in structural complexity than do dendritic networks typical of stream systems. This difference depends partly on how structural complexity is quantified. The effects of network structure on diversity also depend on how diversity itself is calculated. However, the predominate metapopulation approach to habitat network modelling has only calculated diversity in one way. Our results strongly suggest that population processes should be taken into account when examining the effects of habitat network structure on ecological responses.

\section{ACKNOWLEDGEMENTS}

This work builds upon research conducted in eWater CRC project 1A.102. Angus Webb was supported by eWater CRC project Ecological Management and Restoration.

\section{REFERENCES}

Boukal, D. S. and L. Berec (2002). Single-species models of the Allee effect: Extinction boundaries, sex ratios and mate encounters. Journal of Theoretical Biology 218, 375-394.

Diestel, R. (2005). Graph Theory (3rd ed.). Berlin: Springer.

Drechsler, M. and C. Wissel (1997). Separability of local and regional dynamics in metapopulations. Theoretical Population Biology 51, 9-21.

Gaston, K. J., S. L. Chown, and K. L. Evans (2008). Ecogeographical rules: elements of a synthesis. Journal of Biogeography 35, 483-500.

Grant, E. H. C., W. H. Lowe, and W. F. Fagan (2007). Living in the branches: population dynamics and ecological processes in dendritic networks. Ecology Letters 10, 165-175.

Hanski, I. (1998). Metapopulation dynamics. Nature 396, 41-49.

Hanski, I. and O. Ovaskainen (2000). The metapopulation capacity of a fragmented landscape. Nature 404, 755-758.

Kessler, M. (2009). The impact of population processes on patterns of species richness: Lessons from elevational gradients. Basic and Applied Ecology 10, 295-299.

Levin, S. A. (2000). Multiple scales and the maintenance of biodiversity. Ecosystems 3, 498-506.

Levins, R. (1969). Some demographic and genetic consequences of environmental heterogeneity for biological control. Bulletin of the American Entomological Society 15, 237-240.

MacArthur, R. (1957). On the relative abundance of bird species. Proceedings of the National Academy of Sciences of the U.S.A. 43, 293-295.

McGill, B. J. (2010). Towards a unification of unified theories of biodiversity. Ecology Letters 13, 627-642.

Padgham, M. and J. A. Webb (2010). Multiple structural modifications to dendritic ecological networks produce simple responses. Ecological Modelling 221, 2537-2545.

Sahney, S., M. Benton, and P. Ferry (2010). Links between global taxonomic diversity, ecological diversity and the expansion of vertebrates on land. Biology Letters 6, 544-547.

Sayles, R. S. and T. R. Thomas (1978). Surface topography as a nonstationary random process. Nature 271, 431-434.

Sørensen, T. (1948). A method of establishing groups of equal amplitude in plant sociology based on similarity of species content, and its application to analyses of the vegetation on danish commons. Kongelige Danske Videnskabernes Selskabs Biologiske Skrifter 5, 1-34.

Urban, D. L., E. S. Minor, E. A. Treml, and R. S. Schick (2009). Graph models of habitat mosaics. Ecology Letters 12, 260-273.

Whittaker, R. H. (1972). Evolution and measurement of species diversity. Taxon 21, 213-251.

Wiens, J. A. (2002). Riverine landscapes: taking landscape ecology into the water. Freshwater Biology 47, 501-515. 